\title{
Pigmented Villonodular Synovitis as an Atypical Cause of Deep Motor Branch Neuropathy
}

\author{
Jon E. Hammarstedt ${ }^{1}$, Nicholas C. Duethman ${ }^{2}$, David G. Dennison ${ }^{2}$
}

Learning Point of the Article:

This case demonstrates pigmented villonodular synovitis as an unusual cause of ulnar tunnel syndrome at the level of Guyon's canal.

\section{Abstract}

Objectives: Compression of the ulnar nerve in Guyon's canal results in ulnar tunnel syndrome (UTS). The patient may present with sensory and motor deficits (zone 1), motor deficit (zone 2), or sensory deficit (zone 3). The most common causes of UTS include ganglion cysts, idiopathic ulnar nerve compression, occupational pressure neuritis (repetitive compression), prolonged compression, hook of hamate fractures, and arterial thrombus or aneurysm.

Case Report: We report an atypical cause of UTS involving pigmented villonodular synovitis (PVNS) with a review of the literature. Surgical decompression of the ulnar nerve at Guyon's canal has resulted in resolving motor weakness and improved interosseous strength at latest followup.

Conclusion: The most common causes of UTS are ganglion, occupational neuritis, prolonged compression, and ulnar artery thrombi/aneurysms. However, other more rare causes such as PVNS should be considered in the appropriate patient.

Keywords: Neuropathy, ulnar nerve, ulnar tunnel.

\section{Introduction}

The ulnar tunnel, also known as Guyon's canal, is one potential area for compression neuropathy of the ulnar nerve. Compression of the ulnar nerve at the level of the wrist is defined as ulnar tunnel syndrome (UTS) and can result in motor deficits, sensory deficits, or both. The incidence of UTS is not clear, however, the rate is much lower than carpal tunnel syndrome (CTS) [1]. Numerous factors have been described as causing UTS including ganglion cysts, occupational neuritis (repetitive compression), prolonged compression, hook of hamate fractures, ulnar artery thrombus or aneurysm, and repetitive trauma [2].

We present an atypical case of isolated neuropathy of the deep motor branch of the ulnar nerve from pigmented villonodular synovitis (PVNS) and provide findings during the physical exam which help to localize the site of compression within the ulnar tunnel and describe the imaging modalities used to confirm the diagnosis. In this case, the more proximal branch of the ulnar nerve, the branch to abductor digiti minimi, was spared and the patient had strong abduction of the little finger.

\section{Case Report}

A 46-year-old right-handed male presented with a 2-year history of masses on the palmar and dorsal aspect of his right wrist. The masses were firm and did not move with the tendons. The patient reported normal sensation of the hand and denied numbness, tingling and weakness. Physical examination findings are listed in [Table 1]. An MRI of the right wrist was conducted and showed multiple nodules throughout the radiocarpal, intercarpal, distal radioulnar, and CMC joints and the ulnar and carpal tunnels (Fig. 1).

The patient underwent operative excision of the masses using

\begin{tabular}{|c|c|c|c|}
\hline \multirow[b]{2}{*}{ Access this article online } & \multicolumn{3}{|c|}{ Author's Photo Gallery } \\
\hline & & & \\
\hline $\begin{array}{c}\text { Website: } \\
\text { www.jocr.co.in }\end{array}$ & Dr. Jon E. Hammarstedt & Dr. Nicholas C. Duethman & \\
\hline $\begin{array}{l}\text { DOI: } \\
\text { 10.13107/jocr.2021.v11.i04.2162 }\end{array}$ & \multicolumn{3}{|c|}{$\begin{array}{l}\text { 'Department of Orthopaedic Surgery, Allegheny General Hospital, Pittsburgh, PA, } \\
\text { 'Department of Orthopedic Surgery, Mayo Clinic, Rochester, MN } \\
\text { Address of Correspondence: } \\
\text { Dr. Jon Hammarstedt, } \\
\text { Department of Orthopedic Surgery, Allegheny General Hospital, } 1307 \text { Federal Street, 2nd Floor, Pittsburgh, PA } 15212 . \\
\text { E-mail: jon.hammarstedt@gmail.com }\end{array}$} \\
\hline
\end{tabular}

Journal of Orthopaedic Case Reports | pISSN 2250-0685 | eISSN 2321-3817 | Available on www.jocr.co.in | doi:10.13107/jocr.2021.v11.i04.2162 This is an Open Access article distributed under the terms of the Creative Commons Attribution Non-Commercial License (http://creativecommons.org/licenses/by-nc/3.0) which permits unrestricted non-commercial use, distribution, and reproduction in any medium, provided the original work is properly cited. 


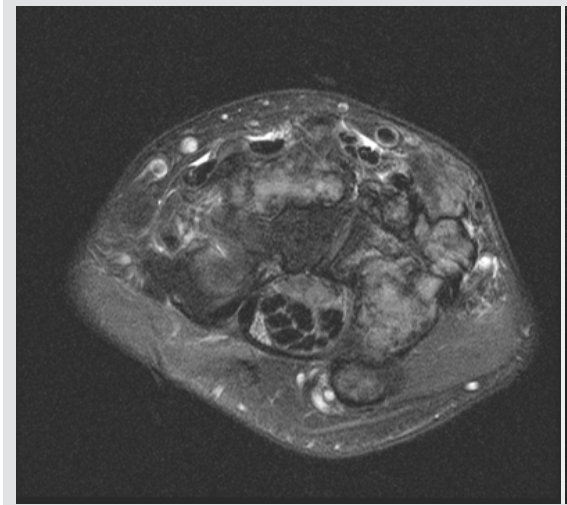

Figure 1: Preoperative plain magnetic resonance imaging of the right wrist of a 46 -year-old male. (A) T2 weighted axia image and (B) T2 weighted coronal image depicting pigmented villonodular synovitis in the volar and dorsal compartments of the wrist compressing the deep motor branch of the ulnad nerve.

volar and dorsal incisions. There were large lobules of a yellow brown mass resected throughout the wrist. A synovectomy and debridement of the radiocarpal, intercarpal, distal radioulnar, and $\mathrm{CMC}$ joints were performed. One large tumor on the floor of the carpal tunnel was resected. All specimens were diagnosed as PVNS. The patient resumed activities after a brief period of immobilization and had an otherwise uneventful recovery.

Three years after the initial surgical excision of PVNS, the patient returned with recurrent nodularity in his wrist and had developed weakness without numbness in his hand. Physical exam findings consisted of intrinsic muscle weakness in the hypothenar and interossei muscles, but intact small finger abduction (Table 1). An MRI was completed and revealed nodules in the carpal tunnel and Guyon's canal (Fig. 2). Electrodiagnostic testing demonstrated a right ulnar nerve mononeuropathy with sparing of the digital branch to the 5th digit.

The patient underwent repeat surgical excision and debridement. Volar and dorsal incisions were used for excision and debulking and intra-operative pathology was again consistent with PVNS (Fig. 3). Tumor was identified and removed from the radiocarpal joint, distal radioulnar joint, the pisotriqueteral joint, multiple intercarpal joints, and within the carpal tunnel. Guyon's canal was explored and revealed a tumor within zone 2 of the ulnar nerve, pressing the nerve against the fibrous arch of the hypothenar muscles (Fig. 4). All visible tumor was resected and the deep motor branch of the ulnar nerve was decompressed. The patient had an uneventful recovery but with improvement in interosseous strength at his recent 3 and 9 -month visit (Table 1).

\section{Discussion}

\section{Ulnar nerve anatomy}

The ulnar nerve enters the hand along with the ulnar artery through Guyon's canal bounded medially by the pisiform and laterally by the hook of the hamate. The branch innervating the abductor digiti minimi most commonly divides proximal to the pisohamate arch and travels superficially to innervate the abductor digiti minimi muscle. Thus, compression of the deep motor branch of the ulnar nerve may be spare the abductor digiti minimi muscle. Lesions to the ulnar nerve near the ulnar tunnel are classified by the location of the lesion [3]. Zone 1 is located proximal to the bifurcation of the ulnar nerve and contains both sensory and motor fibers. Zone 2 includes the deep motor branch of the ulnar nerve and continues deep dorsally and radially and contains motor fibers. Zone 3 is comprised of the superficial branch of the ulnar nerve and continues distally to provide sensory innervation to the medial half of the fourth and entire fifth digit [4].

\section{UTS}

The most common causes of UTS at the wrist are ganglion and

\section{SLIDE DISPOSITION:}

\section{DIAGNOSIS :}

A. Synovium, right wrist, biopsy: Pigmented villonodular synovitis.

This final pathology report is based on the gross/macroscopic examination and the frozen section histologic evaluation of the specimen(s). A revised report will be issued to document the final interpretation after review of the Hematoxylin and Eosin (H\&E) permanent sections.

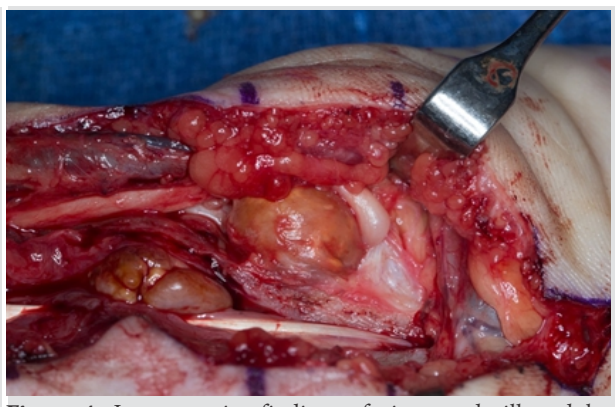

Figure 4: Intraoperative findings of pigmented villonodular synovitis (PVNS) compressing the deep motor branch of the ulnar nerve in a 49-year-old male with PVNS. 
chronic repetitive compression. Ganglion cysts in the ulnar tunnel were first reported by Brooks et al in 1952 and can create compression at any point throughout the tunnel. Similar to ganglion cysts, tumors can cause compression of the ulnar nerve at any point within Guyon's canal, and re-enforces the importance of a good physical exam in localizing the site of compression. Numerous studies have described additional disruptions to the ulnar tunnel consequently affecting the ulnar nerve at each of the three zones $[1,4]$. Fractures of the hook of hamate, pisiform, distal radius, and metacarpals have also been shown to cause UTS. Marie et al. described 47 patients with occupational damage to the hypothenar area due to repetitive "hammering" of the ulnar tunnel causing fibrosis, artery thrombosis, and occlusions [5]. Their cohort experienced symptoms resembling zone 1 lesions. They found favorable outcomes with education and conservative treatment. Ozdemir et al. described a case of ulnar entrapment associated with fibrosis from recurrent CTS affecting zone 1 of the ulnar nerve [6]. The patient underwent additional surgical decompression and experienced clinical improvement in her symptoms. Uysal et al. described a patient with large PVNS tumor causing CTS and symptoms resembling zone 1 UTS [7]. The lesion was excised and the patient had no symptoms at 25 months of follow-up.

The musculature of the hand has also been attributed as a cause of UTS. Hypertrophy of flexor digiti minimi, abductor digiti minimi, aberrant palmaris longus, or palmaris brevis have been shown to cause compression at each of the three zones of the ulnar tunnel [1]. Examiners should be aware of the double crush phenomenon where concomitant compression at the cubital tunnel or vascular compromise renders the distal segment susceptible to injury.

While the annual incidence of UTS is unknown, it is widely considered to be less than CTS and cubital tunnel syndrome. A thoughtful and complete physical exam along with further diagnostic studies are key to localizing the compression and understanding the etiology. Standard radiographs and the carpal tunnel view may help in identifying bony pathology, while magnetic resonance imaging may be used to assess soft tissue and vascular etiology. Electrodiagnostic studies can be performed to localize nerve compression and dysfunction particularly when the physical examination is equivocal. UTS will exhibit normal latencies in the palmar and dorsal cutaneous nerves and proximally innervated muscles.

Treatment should include both non-surgical and surgical options. Conservative treatment should begin with rest, splinting, and anti-inflammatory medications. Activity modifications and physical therapy may also relieve symptoms while preventing progression of symptoms. Surgical management should be aimed at restoration of anatomy and decompression of the ulnar tunnel at all three zones.

\section{PVNS}

PVNS is a benign proliferative disease of the tendon sheath, synovial bursa, and synovial joint with an estimated incidence between 2 and 8 per million per year [8]. PVNS is composed of two unique forms: diffuse and localized. The entire synovium of an affected joint or structure is involved in the diffuse form and is typically pigmented with coarse villi and diffuse nodularity. The localized form involves only a portion of a synovial surface with less pigmentation and villous formation when compared to the diffuse form. Histopathologic evaluation after synovial biopsy is essential to confirm and differentiate the diagnosis.

Preoperative diagnosis of PVNS remains difficult due to the nonspecific presentation and subtle radiographic findings. A thorough history and physical examination including plain radiographs may show bony abnormalities including erosions and cysts [9]. Magnetic resonance imaging is the preferred imaging modality for diagnosis and surgical planning of suspected PVNS. Thorough imaging evaluation of the affected area should be undertaken to assess for asymptomatic lesions.

Diffuse PVNS is predominantly intra-articular affecting the knee joint (up to $75 \%$ ) in individuals younger than 40 years of age. The patient may present with increased pain, decreased range of motion, and swelling. If left untreated, hypertrophic synovium may lead to degenerative changes including joint destruction and osteoarthritis. Diffuse PVNS typically presents as a poorly defined soft tissue mass on magnetic resonance imaging [10]. Localized PVNS predominantly occurs in the hand, knee, and ankle in individuals between 30 and 50 years of age. Patients typically present with a long-standing painless swollen joint, and occasionally localized PVNS is identified as an incidental finding. If untreated, localized PVNS can continue to increase in size causing discomfort and compression of local structures. Localized PVNS presents as a well-circumscribed mass on magnetic resonance imaging. PVNS has been described as both intra- and extra-articular within the hand including the carpal bones, the metacarpophalangeal joints, the phalanges, and the extensor compartments $[11,12,13]$. However, there are no documented reports of PVNS causing isolated entrapment of the deep motor branch of the ulnar nerve (zone2).

Treatment of PVNS depends on the progressive potential of the tumor and consists of observation to surgical resection and medical therapy. Stephan et al. proposed a treatment algorithm that includes tumor type (localized versus diffuse) and progression (stable versus progressive). Stable lesions are 
treated symptomatically with close follow-up while surgical excision is the mainstay for progressive lesions. It is important to perform a capsulotomy to inspect and debride any pigmented tissue within the joint. Recurrence rates for PVNS have been studied to be as high as $29 \%$ with surgical excision alone. External beam radiation therapy has been used as a safe and effective adjuvant therapy for PVNS in the knee to reduce recurrence rates, however, patient numbers are small. Coroneos et al. studied radiation therapy for PVNS in the hand of ten patients and had no recurrence at a mean follow-up of 3.1 years. Medical treatment directed at TNF- $\alpha$, macrophage colonystimulating factor, and tyrosine kinase inhibitors have resulted in prolonged regression and are a potential future treatment modality.

\section{Conclusion}

Our report describes an atypical cause of UTS exclusively involving the deep motor branch of the ulnar nerve and sparing the abductor digiti minimi muscle. Lesions exclusive to zone 3 will present with sensation deficits (numbness or tingling) in the medial half of the fourth digit and the entire 5th digit. Disruptions isolated to zone 2 affect motor fibers and the patients may present with clinical findings such as atrophy of interossei, the inability to cross fingers, and poor key pinch strength via the adductor pollicis (Froment's sign). The patient may exhibit abduction of the small finger and the inability to adduct with the small finger (Wartenburg sign) depending upon the location of the lesion and bifurcation point for the branch innervating the abductor digiti minimi. Compression within zone 1 will present with symptoms of both zone 2 and 3 .

\section{Clinical Message}

Evaluation of UTS includes a history and physical exam with careful attention to sensory and motor deficits. Plain radiographs should include posteroanterior and lateral views. A carpal tunnel view should be obtained if a fracture of the hook of the hamate is suspected although a computed tomography scan or additional cross-sectional imaging should be completed if there is concern for a hook of hamate fracture as well. Magnetic resonance imaging and ultrasound have proven helpful with the identification of soft masses and preoperative planning $[14,15]$. Electromyography may be used to localize the tumor and correlate clinical symptoms with effective comparison to CTS and more proximal compression. The most common causes of UTS are ganglion, occupational neuritis, prolonged compression, and ulnar artery thrombi/aneurysms. However, more rare causes such as PVNS should remain on the differential.

\section{References}

1. Chen SH, Tsai TM. Ulnar tunnel syndrome.J Hand Surg Am 2014;39:571-9.

2. Shea JD, McClain EJ. Ulnar-nerve compression syndromes at and below the wrist. J Bone Joint Surg Am 1969;51:1095103.

3. Gross MS, Gelberman RH. The anatomy of the distal ulnar tunnel. Clin Orthop Relat Res 1985; 196:238-47.

4. Bachoura A, Jacoby SM. Ulnar tunnel syndrome. Orthop Clin North Am 2012;43:467-74.

5. Marie I, Hervé F, Primard E, Cailleux N, Levesque H. Longterm follow-up of hypothenar hammer syndrome: A series of 47 patients. Medicine (Baltimore) 2007;86:334-43.

6. Ozdemir O, Calisaneller T, Gulsen S, Caner H. Ulnar nerve entrapment in Guyon's canal due to recurrent carpal tunnel syndrome: Case report. Turk Neurosurg 2011;21:435-7.

7. Uysal M, Ozkoç G, Akpinar S, Hersekli MA, Tandogan R. Combined ulnar and carpal tunnel syndrome caused by pigmented villo-nodular tenosynovitis: A rare case. Arch Orthop Trauma Surg 2007; 127:563-5.

8. Stephan SR, Shallop B, Lackman R, Kim TW, Mulcahey MK. Pigmented villonodular synovitis: A comprehensive review and proposed treatment algorithm. JBJS Rev 2016;4:01874474-201607000-00005.

9. Bouali H, Deppert EJ, Leventhal LJ, Reeves B, Pope T. Pigmented villonodular synovitis: A disease in evolution.J Rheumatol 2004;31:1659-62.

10. Aurégan JC, Klouche S, Bohu Y, Lefèvre N, Herman S, Hardy P. Treatment of pigmented villonodular synovitis of the knee. Arthroscopy 2014;30:1327-41.

11. Lee YK, Han Y, Lee M. Arthroscopic resection of a tenosynovial giant cell tumor in the Wrist: A case report. Medicine (Baltimore) 2015;94:e1887.

12. Looi KP, Low CK, Yap YM. Pigmented villonodular synovitis of the hand in the Asian population. Hand Surg 1999;4:81-5.

13. Carpintero P, Serrano J, Frasquet GA. Pigmented villonodular synovitis of the wrist invading bone-a report of 2 cases. Acta Orthop Scand 2000;71:424-6.

14. Subin GD, Mallon WJ, Urbaniak JR. Diagnosis of ganglion in Guyon's canal by magnetic resonance imaging. J Hand Surg Am 1989; 14:640-3.

15. Ginanneschi F, Filippou G, Milani P, Biasella A, Rossi A. 
Ulnar nerve compression neuropathy at Guyon's canal caused by crutch walking: Case report with ultrasonographic nerve imaging. Arch Phys Med Rehabil 2009;90:522-4.

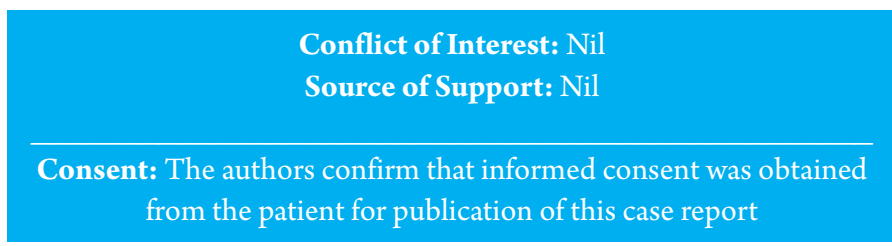

How to Cite this Article

Hammarstedt JE, Duethman NC, Dennison DG. Pigmented Villo Nodular Synovitis as an Atypical Cause of Deep Motor Branch Neuropathy. Journal of Orthopaedic Case Reports 2021 April;11(4):80-84. 